\title{
Non-conservative Management of Chylothorax
}

\author{
Hyo Yeong Ahn, M.D., Hoseok I, M.D.
}

Department of Thoracic and Cardiovascular Surgery, Medical Research Institute, Pusan National University Hospital, Pusan National University School of Medicine, Busan, Korea

\section{ARTICLE INFO}

Received June 3, 2021

Accepted July 2, 2021

Corresponding author

Hoseok I

Tel $82-51-240-7267$

Fax 82-51-243-9389

E-mail ihoseok@pusan.ac.kr

ORCID

https://orcid.org/0000-0001-8930-8148

\begin{abstract}
Chylothorax is caused by lymphatic leakage, which can develop after thoracic surgery and is associated with cancer. Although prospective randomized trials have not been performed, radiological interventions have been performed in several cases with persistent chylothorax, adjunct to 2 weeks of conservative management. The success rate of such interventions is diverse due to anatomical variations, although the results are promising. However, in cases of treatment failure after cycles of interventions, a team approach may be necessary to determine whether surgical management is warranted.
\end{abstract}

Keywords: Chylothorax, Management, Intervention, Surgery

\section{Introduction}

Chylothorax refers to a collection of chyle in the pleural cavity due to leakage from the lymphatic vessels, especially from the thoracic duct. The classification of chylothorax depends on its etiology (traumatic, non-traumatic, or idiopathic). Regardless of the cause, the medical and surgical options to reduce lymph leakage for patients with chylothorax are similar to those for patients with high flow chylothorax ( $>25 \mathrm{~mL} / \mathrm{kg} /$ day for child, $1,000 \mathrm{~mL} /$ day for adult) [1-8]. If even repeated interventions are not effective, surgical treatment might be the last option $[7,9,10]$. In the event that surgical management fails, conservative management may be continued until leakage ceases. Unfortunately, the comparison of outcomes between conservative and surgical treatment is limited by the insufficient number of patients with chylothorax for prospective studies to be conducted $[7,8]$. In this article, the anatomy of the thoracic duct and the etiology, diagnosis, and treatment of chylothorax are discussed.

\section{Anatomy}

The thoracic duct drains the cisterna chyli, located on the right side of the thoracic vertebrae, which continues on the left from the level of T4 and drains into the left innominate vein (Fig. 1). Variations have been observed in more

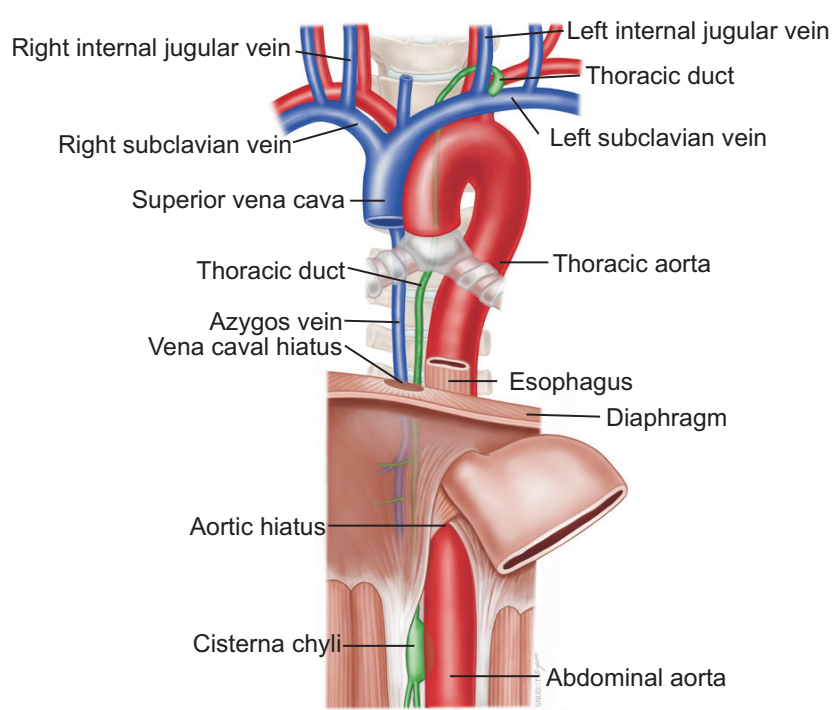

Fig. 1. Anatomy of the thoracic duct. From the cisterna chyli, the thoracic duct crosses the diaphragm at the aortic hiatus at the level of the 12th thoracic vertebra, and continues to ascend between the thoracic aorta on the left and the azygos vein on the right. It crosses over to the left side between the fourth and sixth thoracic vertebrae. Finally, it ascends behind the aortic arch and the left subclavian artery.

than one-third of the population $[2,9,11]$. These anatomic variations are the underlying cause of the diversity of intervention success rates $[2,9,11]$. Moreover, there is a high risk of tearing along the course of the lymphatic pathway 
during esophagectomy and lymph node dissection due to the proximity of the course of the thoracic duct to other structures $[1,12,13]$. Any tear in the thoracic duct can quickly lead to the accumulation of large volumes of lymphatic fluid, since it usually transports around 2-4 L of chyle per day [11].

\section{Etiology}

The etiology of chylothorax can be classified as involving traumatic or non-traumatic injuries to the thoracic duct, although idiopathic chylothorax can also occur $[4,6,14]$.

\section{Traumatic causes (50\%)}

Direct injury to the thoracic duct, particularly during thoracic surgery (e.g., esophagectomy and lymph node dissection), is the most common cause of traumatic chylothorax. Other traumatic causes include blunt trauma or penetrating injuries $[4,6,14]$.

\section{Non-traumatic causes (45\%)}

In cases of non-traumatic chylothorax, direct infiltration or impeded flow caused by compression and accumulation leads to filtration of the lymphatic vessels. Relatively impeded flow can occur if there is increased lymph production, such as in patients with increased central venous pressure or those with liver cirrhosis and portal hypertension. Chylothorax can develop idiopathically in up to $6 \%$ of cases $[4,6,14]$.

\section{Diagnosis}

The presence of chylomicrons, measuring approximately 0.5-1.0 $\mu \mathrm{m}$ in size, needs to be confirmed in the pleural fluid. Alternatively, chylothorax can be established by triglyceride content $>110 \mathrm{mg} / \mathrm{dL}$ and a cholesterol concentration $<200 \mathrm{mg} / \mathrm{dL}$. A triglyceride concentration of $<50 \mathrm{mg} /$ $\mathrm{dL}$ rules out chylothorax. The differential diagnosis includes pseudochylothorax, which is characterized by low triglyceride content, a cholesterol concentration of $>200$ $\mathrm{mg} / \mathrm{dL}$, and the absence of chylomicrons (Fig. 2).

\section{Treatment}

The appropriate treatment method depends on the etiology and amount of chylothorax. Traditionally, conservative treatment is first administered [7,9,13-15]. However, in pa-

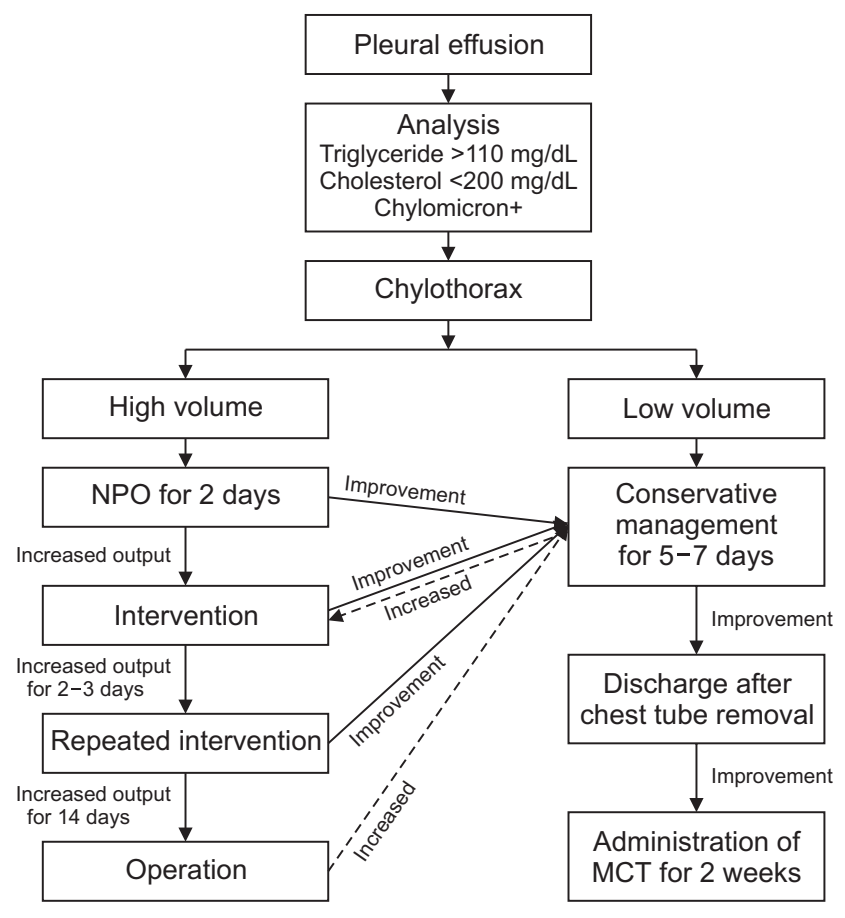

Fig. 2. Algorithm of chylothorax management. NPO, nil per os; $\mathrm{MCT}$, medium-chain triglyceride.

tients with high-volume chylothorax, the timing of appropriate interventions is important. The development of therapeutic methods and an algorithm has ensured a systematic and timely process to deliver treatment (Fig. 2). Further randomized trials need to be conducted to establish a consensus on chylothorax management.

\section{Conservative treatment}

\section{Indications}

Conservative management is considered the first-line modality for all patients with chylothorax [7,9,13-15].

\section{Methods}

Nothing by mouth or a diet containing medium-chain triglycerides (MCT) reduces the flow of lymph and may allow spontaneous closure of the leak in the thoracic duct $[7,9]$. Adequate fluid and electrolyte replacement with proper nutrition should follow. Additionally, pleural drainage may be done for lung re-expansion and pulmonary function optimization. The administration of octreotide may be considered if drain output persists [16].

\section{Prognosis}

The resolution rate after conservative treatment ranges 
from $16 \%$ to more than $75 \%[7,9]$; however, this figure is lower in patients with high-volume chylothorax $(>1,000$ $\mathrm{mL} /$ day). In the event of marked drain output reduction under conservative treatment, a good therapeutic outcome can be achieved after a few weeks of a strict MCT diet.

\section{Interventional treatment}

\section{Indications}

Lymphangiography is advisable for almost all patients with persistent chylothorax for the identification of the level of the tear. This applies for both traumatic and non-traumatic chylothorax $[4,6,11,16-18]$.

\section{Methods}

Resolution of the thoracic duct leak spontaneously occurs in up to $50 \%$ of cases $[4,6,11]$. However, lymphangiography is advisable for the occlusion of chyle fistulas, as has been reported in some cases.

\section{Thoracic duct lymphangiography}

Thoracic duct lymphangiography, which is performed intranodally via the bilateral inguinal or femoral lymph nodes (Fig. 3A-D) $[4,16,18]$, requires a $26 \mathrm{G}$ needle and a short catheter (Cook, Bloomington, IN, USA) fitted to a luer-lock syringe containing 3-5 mL of lipiodol. Ultrasonography and intermittent fluoroscopy are used to confirm intranodal lymphatic vessel puncture and to guide the subsequent injection of lipiodol at $3 \mathrm{~mL} / \mathrm{min}$, respectively. If nodal rupture occurs during the injection, the upper lymph nodes may be directly punctured under fluoroscopic guidance to continue lipiodol administration, which is ceased after the opacification of the lumbar duct or cister- na chyli. The total amount of lipiodol is limited to $20 \mathrm{~mL}$ to minimize the risk of pulmonary fat embolism.

\section{Thoracic duct embolization}

Prior to the procedure, the duct and its adjacent structures (e.g., the aorta) are visualized with cone-beam computed tomography. Thoracic duct embolization is performed by gaining access to the thoracic duct via direct puncture of the lumbar duct or cisterna chyli with a $21 \mathrm{G}$ Chiba needle through the transabdominal route $[2,14,19]$. After transabdominal puncture of the cisterna chyli, a 0.018-inch $(0.46 \mathrm{~mm})$ Transend guidewire (Boston Scientific Corp., Natick, MA, USA) is used to enter the thoracic duct and is advanced further before the introduction of a 2.0F or 2.2F microcatheter (Terumo, Tokyo, Japan), employing an over-the-wire technique (Fig. 3E, F). Digital subtraction angiography, aided with a microcatheter, is used to establish the chyle leakage sites, which are then subjected to Concerto microcoil embolization (Medtronic, Jacksonville, FL, USA) proximal to the leakage sites and embolized using a mixture of $\mathrm{N}$-butyl cyanoacrylate (NBCA, Histoacryl; B Brown, Melsungen, Germany) and lipiodol (weight ratio 1:1). Coil embolization is done to prevent NBCA migration to the left subclavian vein due to the longer NBCA polymerization time in the lymphatic vessels than in the blood vessels. Lipiodol is injected after establishing needle access to the lymph node. Fluoroscopic images of the cisterna chyli are obtained, and under fluoroscopic guidance, the cisterna chyli is directly punctured using a $21 \mathrm{G}$ Chiba needle. A 0.018 -inch $(0.46 \mathrm{~mm})$ guidewire and microcatheter are used to cannulate the thoracic duct, which is then embolized using Concerto 3EA microcoils and an NBCA/lipiodol mixture (weight ratio 1:1).
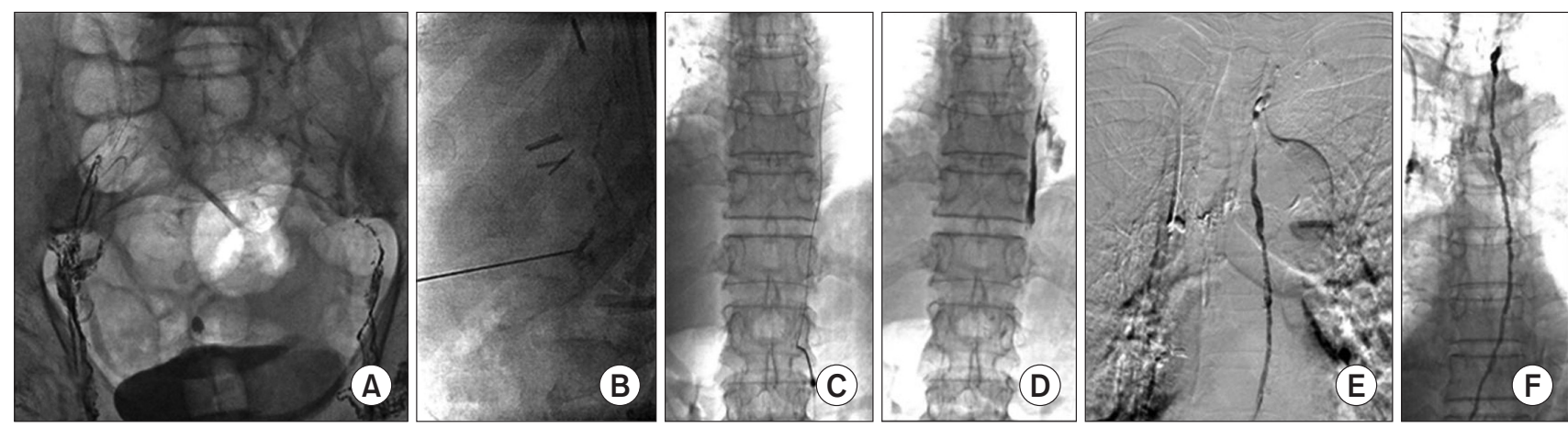

Fig. 3. Thoracic duct embolization. (A) Intranodal lymphangiography via the bilateral inguinal approach, (B) thoracic duct access via direct puncture of the lumbar duct, (C) introduction of a microcatheter after insertion of a guidewire, (D) digital subtraction angiography to define the chyle leakage site, (E) microcoil embolization above the leakage site, and (F) embolization using an N-butyl cyanoacrylate and lipiodol mixture. 
This procedure has a success rate of over $90 \%$ in chylothorax cases $[1,2,4,14,19,20]$.

\section{Needle disruption}

Needle disruption to reduce lymphatic flow may be performed in cases of small prevertebral lymph vessels, which are anatomic variants in $30 \%$ of patients [2].

\section{Transjugular intrahepatic portosystemic stent shunt}

In hepatic chylothorax, transjugular intrahepatic portosystemic shunts may be considered to reduce lymphatic flow [21].

\section{Prognosis}

Due to occurrences of non-traumatic chylothorax in patients with underlying diseases, the success rate of interventions is lower than that of traumatic chylothorax [4]. The reported overall success rate in non-traumatic chylothorax management ranges from $27 \%$ to $68 \%[6,8,11]$. Radiologic intervention might still be considered relatively safe and effective as some studies have reported complication rates of $3 \%$ and no critical complications $[6,8,11]$.

\section{Surgical treatment}

\section{Indications}

Surgery might be the last option if the leak persists for more than 2 weeks, even with conservative treatment or a radiological intervention, or if there is a high-volume chyle leak of more than 1,000-1,500 mL/day [10,21-25].

\section{Surgical methods}

Surgical techniques include thoracic duct ligation, mass ligation of the tissue, pleurodesis, and pleuroperitoneal shunting [10,21-25]. Thoracic duct ligation is performed by ligating or clipping above the right diaphragm, between T8 and T12, regardless of the surgical site (Fig. 4). Preoperative administration of olive oil, ice cream, or heavy cream may be done for better identification of the tear site. Alternatively, mass ligation of tissue around the presumed course of the thoracic duct may be considered. As a way to approach the thoracic duct, direct vision might be generally considered; however, in selected cases, video-assisted thoracic surgery could be another option [21,24,25]. If the aforementioned operations are not practically feasible, pleurodesis may be performed to induce inflammation and subsequent adhesion between the visceral and parietal pleura. If leakage persists after performing the above-mentioned procedures, insertion of a pleuroperitoneal shunt or

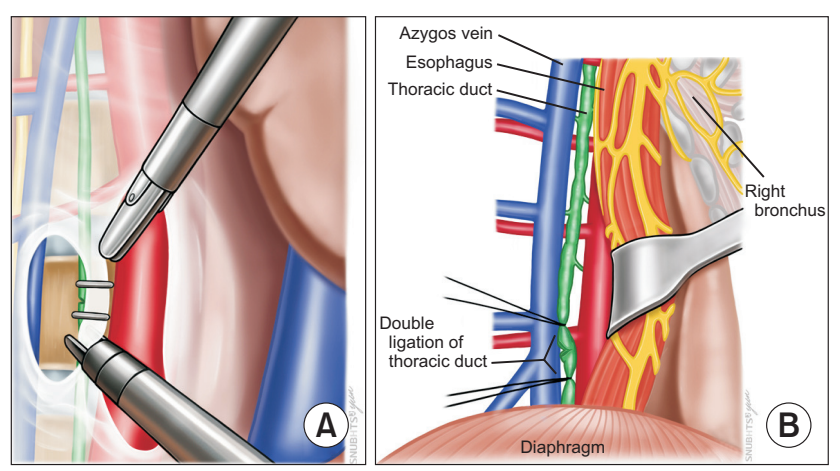

Fig. 4. Surgical technique for the treatment of chylothorax. Thoracic duct ligation can be performed by clipping (A), or ligation (B) above the right diaphragm between T8 and T12.

external permanent drainage should be considered $[10,21$ 25].

\section{Prognosis}

Generally, surgical interventions have a success rate of approximately $90 \%$, with complications occurring in up to $38.3 \%$ of cases [10]. Considerable mortality rates of up to $25 \%$ have been reported $[10,25]$.

\section{Conclusion}

The development of other interventional methods for chylothorax management is limited by individual competency levels. According to the authors' personal observations in the past 4 years, there is a learning curve for the performance of these procedures at our center. Additionally, anatomic variants of the cisterna chyli or lymphatic vessels have fewer puncture sites to access the thoracic duct for intubation. Nevertheless, lymphangiography remains a promising option for leak resolution $[11,18,26]$. This radiological intervention is considered relatively safe and effective, as shown by its success rate of $52 \%$ and complication rate of $3 \%$, without fatal complications $[6,8,11]$.

Although radiological interventions have been performed in several cases of persistent chylothorax, whether the outcomes are successful might be affected by the individual radiologist. Therefore, surgeons should be well informed of the anatomy of the thoracic duct, and a team approach may be necessary to determine whether surgical management is warranted in cases of treatment failure after cycles of intervention. 


\section{Conflict of interest}

No potential conflict of interest relevant to this article was reported.

\section{Acknowledgments}

Figs. 1 and 4 were illustrated by Nayun Lee from medical illustrator, Department of Thoracic and Cardioavascular Surgery, Seoul National University Bundang Hospital.

\section{ORCID}

Hyo Yeong Ahn: https://orcid.org/0000-0003-3198-8237

Hoseok I: https://orcid.org/0000-0001-8930-8148

\section{References}

1. Bazancir LA, Jensen RJ, Frevert SC, Ryom P, Achiam MP. Embolization of the thoracic duct in patients with iatrogenic chylothorax. Dis Esophagus 2021 Feb 8 [Epub]. https://doi.org/10.1093/dote/ doab001.

2. Binkert CA, Yucel EK, Davison BD, Sugarbaker DJ, Baum RA. Percutaneous treatment of high-output chylothorax with embolization or needle disruption technique. J Vasc Interv Radiol 2005;16:1257-62.

3. Borcyk K, Kamil A, Hagerty K, Deer M, Tomich P, Anderson Berry AL. Successful management of extremely high-output refractory congenital chylothorax with chemical pleurodesis using $4 \%$ povidone-iodine and propranolol: a case report. Clin Case Rep 2018;6: 702-8.

4. Hara H, Mihara M, Yamamoto M. Therapeutic lymphangiography for traumatic chylothorax. J Vasc Surg Venous Lymphat Disord 2018; 6:237-40.

5. Jun H, Hur S. Interventional radiology treatment for postoperative chylothorax. Korean J Thorac Cardiovasc Surg 2020;53:200-4.

6. Juszczyk K, Waugh R, Sandroussi C. Lymphangiography as therapeutic management of chylothorax. J Med Imaging Radiat Oncol 2013;57:460-1.

7. Schild HH, Strassburg CP, Welz A, Kalff J. Treatment options in patients with chylothorax. Dtsch Arztebl Int 2013;110:819-26.

8. Schild HH, Pieper C. Chylothorax: current therapeutic options. Zentralbl Chir 2019;144:S24-30.

9. Pulle MV, Puri HV, Asaf BB, Bishnoi S, Yadav A, Kumar A. Chylothorax: modalities of management and outcomes: a case series. Lung India 2021;38:154-60.

10. Tenny BC, Madjarov J, Shipe T. Surgical intervention in a compli- cated persistent chyle leak. Int J Surg Case Rep 2018;42:7-9.

11. Matsumoto T, Yamagami T, Kato $\mathrm{T}$, et al. The effectiveness of lymphangiography as a treatment method for various chyle leakages. Br J Radiol 2009;82:286-90.

12. Tapias LF, Morse CR. A preliminary experience with minimally invasive Ivor Lewis esophagectomy. Dis Esophagus 2012;25:449-55.

13. Varshney VK, Suman S, Garg PK, Soni SC, Khera PS. Management options for post-esophagectomy chylothorax. Surg Today 2021;51: $678-85$.

14. Jeon YJ, Cho JH, Hyun D, et al. Management of chyle leakage after general thoracic surgery: impact of thoracic duct embolization. Thorac Cancer 2021;12:1382-6.

15. Takuwa T, Yoshida J, Ono S, et al. Low-fat diet management strategy for chylothorax after pulmonary resection and lymph node dissection for primary lung cancer. J Thorac Cardiovasc Surg 2013;146:571-4.

16. Sharkey AJ, Rao JN. The successful use of octreotide in the treatment of traumatic chylothorax. Tex Heart Inst J 2012;39:428-30.

17. Nadolski GJ, Itkin M. Lymphangiography and thoracic duct embolization following unsuccessful thoracic duct ligation: imaging findings and outcomes. J Thorac Cardiovasc Surg 2018;156:838-43.

18. Sommer CM, Pieper CC, Itkin M, et al. Conventional lymphangiography $(C L)$ in the management of postoperative lymphatic leakage (PLL): a systematic review. Rofo 2020;192:1025-35.

19. Jayasinghe SA, Srinivasa RN, Hage AN, Gemmete JJ, Majdalany BS, Chick JF. Thoracic duct embolization: analysis of practice patterns. Ann Vasc Surg 2018;52:168-75.

20. Kim PH, Tsauo J, Shin JH. Lymphatic interventions for chylothorax: a systematic review and meta-analysis. J Vasc Interv Radiol 2018;29: 194-202.

21. Lutz P, Strunk H, Schild HH, Sauerbruch T. Transjugular intrahepatic portosystemic shunt in refractory chylothorax due to liver cirrhosis. World J Gastroenterol 2013;19:1140-2.

22. Arshava EV, Parekh KR. Thoracoscopic thoracic duct ligation: how I teach it. Ann Thorac Surg 2020;109:1330-4.

23. Liu Z, Du M, Liang Y, Ju S, Li X, Gao Y. Prophylactic ligation of the thoracic duct branch prevents chylothorax after pulmonary resection for right lung cancer. Surg Today 2020;50:881-8.

24. Yamagata Y, Saito K, Hirano K, Oya M. Laparoscopic transhiatal thoracic duct ligation for chylothorax after esophagectomy. Thorac Cardiovasc Surg 2019;67:606-9.

25. Yang RF, Liu TT, Wang P, et al. Ligation of thoracic duct during thoracoscopic esophagectomy can lead to decrease of $T$ lymphocyte. $\mathrm{J}$ Cancer Res Ther 2018;14:1535-9.

26. Yamagami T, Masunami T, Kato T, et al. Spontaneous healing of chyle leakage after lymphangiography. Br J Radiol 2005;78:854-7. 